\title{
PERCEPCIÓN DE PROFESIONALES EN ADMINISTRACIÓN SOBRE ALGUNAS VARIABLES ORGANIZACIONALES COMO POTENCIALES GENERADORES DE ESTRÉS LABORAL
}

\author{
DIEGO J. QUIRÓS MORALES \\ Universidad Fidélitas, Costa Rica \\ djquiros@gmail.com
}

\section{RESUMEN}

La incapacidad de respuesta ante demandas organizacionales producto de su ejercicio puede derivar en factores de riesgo psicosocial tales como el estrés. El presente estudio tuvo como objetivo la valoración de la percepción de profesionales de la administración y los negocios sobre algunas variables organizacionales como potenciales generadores de estrés laboral. Se diseñó y aplicó el Cuestionario para la Valoración de Factores Generadores de Estrés en las Organizaciones (CUVEO). El instrumento fue aplicado en una muestra de 784 sujetos masculinos y femeninos entre los 19 y los 70 años de edad, la mayoría con universidad completa. Los datos fueron analizados mediante el software SPSS. En términos generales, la valoración de la percepción de los participantes sobre las variables indagadas como potenciales generadores de estrés ha resultado en un nivel medio, por lo que refiere a la importancia de la atención de su alcance.

PALABRAS CLAVE: ESTRÉS LABORAL, CONDICIONES DE TRABAJO, DEMANDAS ORGANIZACIONALES.

\section{ABSTRACT}

The inability to respond to organizational demands resulting from its exercise can lead to psychosocial risk factors such as stress. The objective of this study was to assess the perception of management and business professionals about some organizational variables as potential sources of work stress. The Questionnaire for the assessment of factors that generate stress in organizations (CUVEO) was designed and applied. The instrument was applied in a sample of 784 male and female subjects between 19 and 70 years of age with minimal schooling of incomplete university (Most of them with complete college) The data was analyzed using the SPSS software (for its acronym in English). In general terms, the assessment of the participants' perception of the variables investigated as potential generators of stress has resulted in a medium level, which refers to the importance of attention to their scope.

KEY WORDS: WORK STRESS, WORKING CONDITIONS, ORGANIZATIONAL DEMANDS. 


\section{INTRODUCCIÓN}

En la actualidad, la subsistencia de las organizaciones que dependen de una rentabilidad se basa principalmente en su capacidad de competir (Unger, 2018). En un mercado de competencia se consideran tanto factores extrínsecos (entorno general y específico, así como la contribución del medio local a los resultados empresariales) como intrínsecos (a través componentes tales como negocio, soporte, cultura y procesos) (Chiavenato, 2009).

Se han utilizado diferentes estrategias por parte de los responsables de las organizaciones para alcanzar niveles competitivos. Jiménez (2006) hace referencia a elementos tales como la reducción de costos, despido de trabajadores, presión a la fuerza de ventas, ahorro en todas las áreas de la empresa, hasta la implantación de nuevos modelos de administración y gestión empresarial, las cuales son dependientes de la conducta de los trabajadores.

Los trabajadores, como seres sociales, se ven permeados por las condiciones ambientales que las organizaciones ofrecen. Dependiendo de la organización, su estructura y enfoque de productividad, estas condiciones serán generadoras de reacción en los individuos. A su vez, las reacciones pueden ser positivas, favoreciendo la competitividad y rentabilidad, o negativas, generando múltiples posibles contingencias, dentro de las cuales se encuentra el estrés (Carrión, Gutiérrez y López, 2014).

El estrés es un proceso que se inicia ante un conjunto de demandas ambientales que recibe el individuo, a las cuales se debe dar una respuesta adecuada, poniendo en marcha sus recursos de afrontamiento (Robbins y Judge, 2013). Cuando la demanda del ambiente es equivalente a los recursos de afrontamiento que se poseen, se van a desarrollar una serie de reacciones adaptativas de movilización de recursos que implican activación conductual. Al contrario, la incapacidad de respuesta ante estas demandas puede derivar en factores de riesgo psicosocial tales como el estrés (Moreno y Báez, 2010). En esta línea, el estrés sería caracterizado como un elemento obstaculizador, impidiendo al trabajador ser eficaz y competente en el trabajo (Podsakoff, Lepine, y Lepine, 2007). Según Robbins y Judge (2013), niveles de estrés inadecuados son una de las principales fuentes generadoras de cambios a nivel de variables tales como rotación, ausentismo, productividad y satisfacción; todas estas impactan en el correcto funcionamiento de las organizaciones.

Existen estudios que han evidenciado diversos predictores desencadenantes de estrés laboral; por ejemplo, en casos donde se perciben, por parte del trabajador, altos niveles de presión en el trabajo y falta de apoyo social y malestares subjetivos en salud (Sterud, Hem, Lau, y Ekeberg, 2011). Asimismo, variables como la administración de cargas laborales, insuficiencia de insumos para laborar, ausencia de comunicación y estilos de gestión, entre otros, han demostrado ser elementos generadores de estrés (Assadi, 2003).

Se ha encontrado evidencia que refiere a la edad como un elemento de potencial estrés laboral; se ha encontrado que, conforme incrementa la edad, se reportan mayores índices de estrés laboral (Ahola, Honkonen, Virtanen, Aromaa, y Lönnqvist, 2008). Además, se ha relacionado a una vida familiar y social inestable una serie de cambios y alteraciones (síntomas de insomnio, agotamiento emocional, despersonalización) en el ámbito laboral a lo largo de la vida del trabajador (Gencay y Gencay, 2011; Torres, Lambert, Lawver, 2010).

Para interpretar adecuadamente la relación entre las variables mencionadas y el contexto laboral, debe tenerse presente que las empresas cambian de manera constante, operativa o estructuralmente, sea para responder a las necesidades del mercado o para adecuarse a los cambios. Estos cambios, dependiendo de la capacidad 
de afrontamiento del individuo, pueden afectar el desempeño y productividad. Lo anterior refiere a la importancia del objetivo del presente estudio: valorar la percepción de profesionales de la administración y los negocios sobre algunas variables organizacionales como potenciales generadores de estrés laboral, con miras a tener insumos preventivos que anticipen consecuencias que se contrapongan con los objetivos individuales y organizacionales.

\section{METODOLOGÍA}

\section{TIPO DE ESTUDIO}

La investigación se enmarcó dentro de un estudio con alcance descriptivo y fue de tipo cuantitativo.

\section{PARTICIPANTES}

Participaron del estudio un total de 784 sujetos; todos profesionales de la administración y los negocios, según Clasificación de Ocupaciones de Costa Rica (INEC, 2013). De ellos, la mayoría cuenta con universidad completa (92\% de los participantes) o con un grado académico mínimo de universidad incompleta (8\%); en pleno ejercicio profesional y con un mínimo de 6 meses de pertenecer a la organización para la cual laboran. En la tabla 1 se detallan estas características sociodemográficas.

\section{INSTRUMENTO}

Para efectos de la medición se utilizó el Cuestionario para la Valoración de Factores Generadores de Estrés en las Organizaciones (CUVEO), elaborado por el autor del presente escrito (ver Apéndice). La herramienta final está constituida por 42 ítems con 5 opciones de respuesta tipo Likert como unidad de medición: nunca; casi nunca; algunas veces; casi siempre y siempre. Se distribuye en 7 variables: 1) liderazgo; 2) estructura organizacional; 3) demandas de rol;
4) desarrollo laboral; 5) demandas interpersonales; 6) demandas de la tarea y 7) condiciones de trabajo (ver distribución en tabla 2).

\section{DEFINICIÓN DE VARIABLES}

\section{- $\quad$ Liderazgo}

Está asociada a la confianza que inspira el líder en sus seguidores. Aquí se valora la comunicación de las jefaturas, el respeto en cuanto a relaciones de trabajo, orientación, el sentimiento de preocupación por el bienestar que los colaboradores perciben del nivel superior y la confianza por el trabajo realizado (García-Villamizar, 2007). Considera autonomía, procedimientos para desempeñar el trabajo y métodos de evaluación (Gil-Monte, 2011).

\section{- Estructura organizacional}

Refiere a cómo se dividen, agrupan y coordinan formalmente las tareas de trabajo (Robbins y Judge, 2013). Considera elementos tales como: la organización de trabajo, las normas y reglas de gestión, la responsabilidad, la comunicación y procesos de decisión (Chiavenato, 2009). Refiere, también, a elementos tales como herramientas necesarias para desarrollo de sus funciones (García-Villamizar, 2007), flexibilidad, capacidad de respuesta ante las innovaciones, problemas en la incorporación de innovaciones y la disponibilidad de la tecnología para agilizar el trabajo (Gil-Monte, 2011). Requiere de un puesto bien definido como modelo de acción (Newstrom, 2011).

\section{- Demandas del rol}

Se relacionan con las presiones que sufre una persona como función del rol particular que desempeña en la organización. La ambigüedad del rol se crea cuando las expectativas de este no se 
TABLA 1

VARIABLES SOCIODEMOGRÁFICAS DE LOS PARTICIPANTES DEL ESTUDIO

\begin{tabular}{|c|c|c|c|}
\hline Variable & Categoría & Frecuencia & Porcentaje \\
\hline \multirow{2}{*}{ Sexo } & Femenino & 474 & 60 \\
\hline & Masculino & 310 & 40 \\
\hline \multirow{4}{*}{ Edad } & 19a 31 años & 158 & 20 \\
\hline & 32 a 40 años & 210 & 27 \\
\hline & 41 a 50 años & 228 & 29 \\
\hline & 51 a 70 años & 188 & 24 \\
\hline \multirow{3}{*}{ Etado Civil } & Casado / unión libre & 468 & 60 \\
\hline & En una relación de pareja (no viven juntos) & 39 & 5 \\
\hline & Soltero / viudo / divorciado & 277 & 35 \\
\hline \multirow{5}{*}{ Hijos } & 1 hijo & 172 & 22 \\
\hline & 2 hijos & 216 & 28 \\
\hline & 3 hijos & 84 & 11 \\
\hline & 4 a 6 hijos & 31 & 4 \\
\hline & No & 281 & 36 \\
\hline \multirow{2}{*}{ Escolaridad } & Universidad completa & 724 & 92 \\
\hline & Universidad incompleta & 60 & 8 \\
\hline \multirow{2}{*}{ Naturaleza de la organización } & Privada & 230 & 29 \\
\hline & Pública & 554 & 71 \\
\hline \multirow{2}{*}{ Condición del puesto } & Permanente (Propiedad) & 672 & 86 \\
\hline & Por tiempo definido (Interino) & 112 & 14 \\
\hline \multirow{4}{*}{ Antigüedad en la organización } & $0.5-5$ años & 192 & 24 \\
\hline & 10.1-20 años & 215 & 27 \\
\hline & 20.1 a 60 años & 202 & 26 \\
\hline & 5.1 a 10 años & 175 & 22 \\
\hline
\end{tabular}

Fuente: Elaboración propia. $N=784$

TABLA 2

DISTRIBUCIÓN DE ÍTEMS POR VARIABLE DEL CUVEO

\begin{tabular}{|c|c|}
\hline \multicolumn{1}{|c|}{ Variables } & Ítems \\
\hline Liderazgo & $8,17,23,30,34,38,32,35,39$ \\
\hline Estructura organizacional & $5,14,20,27,36,40,28,7,16,22$, \\
& $4,37,42$ \\
\hline Demandas de rol & $26,33,41,25,13,31$ \\
\hline Desarrollo laboral & $9,18,24$ \\
\hline Demandas interpersonales & $3,12,19,29$ \\
\hline Demandas de la tarea & $1,10,2,11$ \\
\hline Condiciones de trabajo & $6,15,21$ \\
\hline
\end{tabular}

Fuente: Elaboración propia.

32 10(1), Enero - Junio, 2019 
entienden con claridad y el trabajador no está seguro de lo que hace (Robbins \& Judge, 2013).

Considera límites de sus competencias y expectativas de los miembros del conjunto de rol sobre su desempeño (Gil-Monte, 2011), así como la definición de parámetros para el ejercicio de una tarea y la capacitación que recibe para efectos del cumplimiento de la tarea, incluyendo habilidades tecnológicas.

\section{- Desarrollo laboral}

Considera elementos tales como el cambio de puesto, las promociones y el desarrollo y las transiciones de carrera, oportunidades de capacitación (Peiró, 1999; García-Villamizar, 2007).

\section{- Demandas interpersonales}

Son presiones creadas por otros empleados. La falta de apoyo social de los colegas, la incompetencia profesional y las malas relaciones interpersonales (Robbins y Judge, 2013). Considera honradez, reciprocidad y equidad en sus intercambios sociales (Gil-Monte, 2011) (ver tabla 6).

\section{- Demandas de la tarea}

Son factores relacionados con el trabajo de una persona (Robbins y Judge, 2013). Entre ellos se encuentran la participación en la toma de decisiones, complejidad de la tarea, presión y sobrecarga del rol de trabajo.

\section{- Condiciones de trabajo}

Considera condiciones de trabajo y distribución física del sitio (Robbins y Judge, 2013). Refiere a elementos tales como temperatura, ruido e iluminación en el espacio de trabajo (GarcíaVillamizar, 2007).

\section{CRITERIOS DE CALIFICACIÓN DEL CUVEO}

Para efectos de la calificación de los puntajes obtenidos por la muestra en estudio, se procedió a la definición de los baremos de calificación del instrumento, bajo los siguientes pasos:

1. Se realizó la suma total y por variable de los puntajes directos obtenidos a partir de las respuestas de los participantes del estudio.

2. Los valores resultantes fueron convertidos en puntajes estandarizados (puntajes Z), los cuales indican cuántas unidades de desviación estándar del promedio está un puntaje determinado.

3. Se calculó la probabilidad acumulada de la distribución de los valores estandarizados (CDF por sus siglas en inglés).

4. Se definieron los decatipos a partir de la multiplicación del percentil por el decatipo definido $(10,20,30,40,50,60,70,80$ y 90$)+1$.

5. Se ordenaron los casos en función del rango definido para cada decatipo a partir del puntaje directo alcanzado (ver tabla 3).

La correcta interpretación indica que todo valor ubicado en el decatipo 5 presenta una puntuación promedio en cuanto a la percepción de las variables organizacionales incluidas en el CUVEO como potenciales generadores de estrés. Todo puntaje ubicado en los decatipos 4, 3, 20 1, indica que las variables organizaciones medidas son percibidas como estresantes por debajo del promedio, lo cual es un aspecto positivo en el marco del presente estudio y herramienta diseñada. Los puntajes que se ubican en los decatipos 6, 7, 8, 9 o 10 son clasificados como puntajes por encima del promedio de respuesta; indicarían que existe un efecto adverso en cuanto a la percepción del individuo por sobre la variable específica o nivel general, a mayor nivel mayor proximidad con una percepción crítica por parte del individuo ante las variables medidas. La interpretación general se expresa en la tabla 4. 
TABLA 3

PUNTUACIONES DIRECTAS MÍNIMAS Y MÁXIMAS POR DECATIPO Y POR VARIABLE

\begin{tabular}{|c|c|c|c|c|c|c|c|c|c|c|c|c|c|c|c|c|c|c|c|c|}
\hline \multicolumn{21}{|c|}{ Decatipo $^{\mathrm{a}}$} \\
\hline & \multicolumn{2}{|c|}{1} & \multicolumn{2}{|c|}{2} & \multicolumn{2}{|c|}{3} & \multicolumn{2}{|c|}{4} & \multicolumn{2}{|c|}{5} & \multicolumn{2}{|c|}{6} & \multicolumn{2}{|c|}{7} & \multicolumn{2}{|c|}{8} & \multicolumn{2}{|c|}{9} & \multicolumn{2}{|c|}{10} \\
\hline & - & + & - & + & - & + & - & + & - & + & - & + & - & + & - & + & - & + & - & + \\
\hline Liderazgo & 0 & 1 & 2 & 5 & 6 & 8 & 9 & 11 & 12 & 14 & 15 & 16 & 17 & 19 & 20 & 22 & 23 & 26 & 27 & 36 \\
\hline Estructura Organizacional & 0 & 8 & 9 & 13 & 14 & 17 & 18 & 20 & 21 & 23 & 24 & 27 & 28 & 30 & 31 & 34 & 35 & 39 & 40 & 52 \\
\hline Demandas de Rol & 0 & 0 & 1 & 3 & 4 & 5 & 6 & 6 & 7 & 8 & 9 & 9 & 10 & 11 & 12 & 13 & 14 & 15 & 16 & 24 \\
\hline Desarrollo Laboral & 0 & 0 & 1 & 2 & 3 & 3 & 4 & 5 & 6 & 6 & 7 & 7 & 8 & 8 & 9 & 9 & 10 & 11 & 12 & 12 \\
\hline Demandas Interpersonales & 0 & 1 & 2 & 3 & 4 & 4 & 5 & 5 & 6 & 6 & 7 & 7 & 8 & 8 & 9 & 10 & 11 & 11 & 12 & 16 \\
\hline Demandas de la Tarea & 0 & 2 & 3 & 4 & 5 & 5 & 6 & 6 & 7 & 7 & 8 & 8 & 9 & 9 & 10 & 10 & 11 & 11 & 12 & 16 \\
\hline Condiciones de Trabajo & 0 & 0 & 1 & 1 & 2 & 2 & 3 & 3 & 4 & 4 & 5 & 5 & - & - & 6 & 6 & 7 & 8 & 9 & 12 \\
\hline Índice General & 0 & 26 & 27 & 42 & 43 & 52 & 53 & 62 & 63 & 71 & 72 & 79 & 80 & 89 & 90 & 99 & 100 & 115 & 116 & 160 \\
\hline
\end{tabular}

Fuente: Elaboración propia.

TABLA 4

CLASIFICACIÓN DE LOS PUNTAJES A PARTIR DE LA PERCEPCIÓN DEL INDIVIDUO SOBRE LAS VARIABLES EN ESTUDIO

\begin{tabular}{|c|c|}
\hline Clasificación & Puntaje \\
\hline Nulo & Puntajes ubicados en decatipo 1 \\
\hline Bajo & Puntajes ubicados en decatipo 2 y 3 \\
\hline Medio bajo & Puntajes ubicados en decatipo 3 y 4 \\
\hline Medio & Puntajes ubicados en decatipo 5 \\
\hline Medio alto & Puntajes ubicados en decatipo 6 y 7 \\
\hline Alto & Puntajes ubicados en decatipo 8 y 9 \\
\hline Crítico & Puntajes ubicados en decatipo 10 \\
\hline
\end{tabular}

Fuente: Elaboración propia.

\section{PROCEDIMIENTO}

La escala fue desarrollada por el autor del presente escrito (ver Apéndice). Los pasos para su construcción fueron los siguientes:

1. Se determinaron los factores teóricos a evaluar.

2. Se derivaron los principales indicadores obtenidos de la literatura nacional e internacional.

3. Una vez determinados los indicadores, se procedió a redactar los ítems correspondientes. Estos fueron adecuados a las características lingüísticas de la muestra de personas con las que se iba a trabajar.
4. Los ítems fueron sometidos a la revisión de profesionales expertos en el tema, quienes tenían conocimiento sobre las características de la población meta. De acuerdo con las valoraciones y recomendaciones que brindaron, se modificó la redacción y las características de algunos ítems.

5. La escala fue sometida a estudio piloto, se hizo la corrección final y se realizaron ajustes en los ítems de acuerdo con los patrones estadísticos obtenidos en esta fase.

6. El CUVEO final fue aplicado a una población de 784 sujetos. El formulario fue enviado vía correo electrónico mediante la comunicación del 
Colegio Profesional de Ciencias Económicas de Costa Rica.

7. Los datos recibidos fueron analizados y procesados mediante el Paquete Estadístico para las Ciencias Sociales (SPSS, por sus siglas en inglés) versión 21.0.

\section{ESTADÍSTICOS}

Levene: la prueba fue utilizada para determinar la homogeneidad de la varianza de la muestra. Fue aplicada a un índice general y por agrupación de variable.

Alfa de Cronbach (a): se utilizó con el fin de evaluar la confiabilidad del instrumento y las variables que la componen.

Análisis de factores: se utilizó el método de máxima verosimilitud y con rotación varimax. Este tipo de rotación fue elegida debido a que dispersa la mayor cantidad de varianza a través de los factores $y$, al mismo tiempo, trata de obtener una estructura simple (Kerlinger y Lee, 2002), lo cual aporta a la estrategia de análisis elegida para valorar el instrumento en estudio. Con la finalidad de seleccionar e interpretar los factores integrantes, se tomó como criterio de elección a aquellos cuyos autovalores ("eigenvalues" en inglés) fueran $\geq 1$ y que tuvieran al menos dos reactivos por factor. De igual forma, para poder elegir a un reactivo como perteneciente a un factor, se tomó como criterio de selección que su carga factorial (alpha) fuera \pm 0.40 (Pérez, 2004).

Prueba de Kaiser, Meyer y Olkin (KMO): se utilizó para comparar las magnitudes de las correlaciones observadas con las correlaciones parciales. Considera valores de 0 a 1, valores inferiores a .5 se consideran inadecuados, aquellos superiores a .5 serán aceptables. Mientras más cercanos estén los valores a 1, mejor es la adecuación de los datos al modelo factorial. La prueba de esfericidad de Bartlett busca rechazar la hipótesis nula de variables iniciales intercorrelacionadas (Pérez, 2004).
Coeficiente de correlación producto momento de Pearson (r): se aplicó con el fin de establecer la relación entre las variables del instrumento.

Anova de 1 vía: con el objetivo de evaluar si existe una diferencia significativa entre las medias de las variables a partir de su clasificación por variables sociodemográficas.

\section{RESULTADOS}

Previo a la presentación de los resultados alcanzados a partir de la aplicación del instrumento diseñado y aplicado, se procedió al análisis de las características del instrumento, con el objetivo de validar la fuente de los hallazgos acá expresos. Se determinó que la escala presenta una distribución homogénea a partir de su índice general y bajo la distribución por género (ver tabla 5).

En cuanto a los niveles de confiabilidad, el nivel alcanzado por el CUVEO fue $a=.96$. El análisis por variable evidenció puntajes de confiabilidad estadísticamente aceptables en todas las agrupaciones (ver tabla 6).

Se aplicó un análisis de factores sobre los 42 ítems de la herramienta y se confirmó estadísticamente la distribución teórica del CUVEO. Se determinó que las variables incluidas en la matriz de correlación presentaban valores apropiados para la realización de un análisis de factores (KMO = .95) y no se encontraban inter-correlacionadas (Bartlett $\left.=X 2_{(861)}=25328.10 ; p<.0001\right)$. Los primeros siete factores explicaron un $62.41 \%$ de la varianza acumulada. La tabla 7 describe las raíces factoriales y varianzas de cada factor dentro de la escala y en la tabla 8 se exponen los ítems resultantes del análisis de factores con sus respectivas cargas factoriales.

El análisis de correlación producto momento de Pearson evidenció relaciones significativas, tanto entre los índices de las variables del instrumento y el índice general (ver tabla 9). 


\section{TABLA 5}

HOMOGENEIDAD POR FACTOR Y TOTAL DEL CUVEO

\begin{tabular}{|c|c|}
\hline \multicolumn{1}{|c|}{ Variable } & Prueba Levene; Significancia $(\boldsymbol{p})$ \\
\hline Liderazgo & Levene $(1,782)=1.12 ; p<.28$ \\
\hline Estructura Organizacional & Levene $(1,782)=0.26 ; p<.60$ \\
\hline Demandas de Rol & Levene $(1,782)=0.04 ; p<.83$ \\
\hline Desarrollo Laboral & Levene $(1,782)=2.04 ; p<.15$ \\
\hline Demandas Interpersonales & Levene $(1,782)=0.37 ; p<.54$ \\
\hline Demandas de la Tarea & Levene $(1,782)=0.17 ; p<.67$ \\
\hline Condiciones de trabajo & Levene $(1,782)=0.04 ; p<.83$ \\
\hline Índice General & Levene $(1,782)=0.28 ; p<.59$ \\
\hline
\end{tabular}

Fuente: Elaboración propia.

TABLA 6

CONFIABILIDAD POR FACTOR Y TOTAL DEL CUVEO

\begin{tabular}{|c|c|}
\hline Variable & Alpha de Cronbach (a) \\
\hline Liderazgo & $a=.92$ \\
\hline Estructura Organizacional & $a=.92$ \\
\hline Demandas de Rol & $a=.86$ \\
\hline Desarrollo Laboral & $a=.90$ \\
\hline Demandas Interpersonales & $a=.80$ \\
\hline Demandas de la Tarea & $a=.70$ \\
\hline Condiciones de trabajo & $a=.75$ \\
\hline Índice General & $a=.96$ \\
\hline
\end{tabular}

Fuente: Elaboración propia.

TABLA 7

RAÍCES FACTORIALES Y VARIANZAS DEL CUVEO

\begin{tabular}{|c|c|c|c|}
\hline \multicolumn{1}{|c|}{ Factor } & Autovalores & \% varianza & \% varianza acumulada \\
\hline Liderazgo & 16.25 & 38.71 & 38.71 \\
\hline Estructura Organizacional & 2.11 & 5.04 & 43.75 \\
\hline Demandas de Rol & 1.90 & 4.53 & 48.29 \\
\hline Desarrollo Laboral & 1.73 & 4.13 & 52.42 \\
\hline Demandas Interpersonales & 1.60 & 3.81 & 56.24 \\
\hline Demandas de la Tarea & 1.33 & 3.17 & 59.41 \\
\hline Condiciones de trabajo & 1.25 & 2.99 & 62.41 \\
\hline
\end{tabular}

Fuente: Elaboración propia. 
TABLA 8

ÍTEMS RESULTANTES DEL ANÁLISIS DE FACTORES CON SUS RESPECTIVAS CARGAS FACTORIALES

\begin{tabular}{|c|c|c|c|c|c|c|c|}
\hline \multicolumn{8}{|c|}{ Factor } \\
\hline Ítem & $\begin{array}{c}1 \\
\text { Liderazgo }\end{array}$ & $\begin{array}{c}2 \\
\text { Estructura } \\
\text { Organizacional }\end{array}$ & $\begin{array}{c}3 \\
\text { Demandas } \\
\text { de Rol }\end{array}$ & $\begin{array}{c}4 \\
\text { Desarrollo } \\
\text { Laboral }\end{array}$ & $\begin{array}{c}5 \\
\text { Demandas } \\
\text { interperso- } \\
\text { nales }\end{array}$ & $\begin{array}{c}6 \\
\text { Demandas } \\
\text { de la Tarea }\end{array}$ & $\begin{array}{c}7 \\
\text { Condiciones } \\
\text { de Trabajo }\end{array}$ \\
\hline 34 & 0.765 & & & & & & \\
\hline 35 & 0.731 & & & & & & \\
\hline 38 & 0.708 & & & & & & \\
\hline 17 & 0.698 & & & & & & \\
\hline 30 & 0.669 & & & & & & \\
\hline 39 & 0.628 & & & & & & \\
\hline 23 & 0.583 & & & & & & \\
\hline 8 & 0.578 & & & & & & \\
\hline 32 & 0.570 & & & & & & \\
\hline 16 & & 0.782 & & & & & \\
\hline 37 & & 0.746 & & & & & \\
\hline 40 & & 0.681 & & & & & \\
\hline 5 & & 0.640 & & & & & \\
\hline 36 & & 0.616 & & & & & \\
\hline 14 & & 0.594 & & & & & \\
\hline 7 & & 0.565 & & & & & \\
\hline 20 & & 0.562 & & & & & \\
\hline 27 & & 0.553 & & & & & \\
\hline 28 & & 0.502 & & & & & \\
\hline 22 & & 0.500 & & & & & \\
\hline 4 & & 0.482 & & & & & \\
\hline 42 & & 0.385 & & & & & \\
\hline 33 & & & 0.783 & & & & \\
\hline 13 & & & 0.679 & & & & \\
\hline 26 & & & 0.640 & & & & \\
\hline 25 & & & 0.554 & & & & \\
\hline 41 & & & 0.524 & & & & \\
\hline 31 & & & 0.469 & & & & \\
\hline 9 & & & & 0.842 & & & \\
\hline 18 & & & & 0.837 & & & \\
\hline 24 & & & & 0.771 & & & \\
\hline
\end{tabular}




\begin{tabular}{|c|c|c|c|c|c|c|c|}
\hline Ítem & $\begin{array}{c}1 \\
\text { Liderazgo }\end{array}$ & $\begin{array}{c}2 \\
\text { Estructura } \\
\text { Organizacional }\end{array}$ & $\begin{array}{c}3 \\
\text { Demandas } \\
\text { de Rol }\end{array}$ & $\begin{array}{c}4 \\
\text { Desarrollo } \\
\text { Laboral }\end{array}$ & $\begin{array}{c}5 \\
\text { Demandas } \\
\text { interpersonales }\end{array}$ & $\begin{array}{c}6 \\
\text { Demandas } \\
\text { de la Tarea }\end{array}$ & $\begin{array}{c}7 \\
\text { Condiciones } \\
\text { de Trabajo }\end{array}$ \\
\hline 19 & & & & & 0.756 & & \\
\hline 12 & & & & & 0.666 & & \\
\hline 3 & & & & & 0.665 & & \\
\hline 29 & & & & & 0.598 & & \\
\hline 2 & & & & & & 0.719 & \\
\hline 1 & & & & & & 0.713 & \\
\hline 11 & & & & & & 0.607 & \\
\hline 10 & & & & & & 0.558 & \\
\hline 6 & & & & & & & 0.743 \\
\hline 15 & & & & & & & 0.730 \\
\hline 21 & & & & & & & 0.730 \\
\hline
\end{tabular}

Fuente: Elaboración propia.

Se procedió con la calificación de la herramienta en función de la baremación definida en la metodología. Para ello, se establecieron los puntajes mínimos y máximos de cada variable y total, así como su respectiva media y desviación típica, todos en función de sus puntuaciones directas (ver tabla 10).

En términos generales, todas las variables (incluido el índice general) se ubicaron en un nivel medio en cuanto a si se percibían como potenciales generadores de estrés, exceptuando las variables Estructura organizacional y Demandas interpersonales, cuyos puntajes alcanzaron un nivel medio alto. Al realizar el análisis de los puntajes alcanzados a partir de las variables sociodemográficas de los participantes del estudio, no se encontraron variantes en los puntajes en función del sexo, estado civil, escolaridad y condición del puesto.

En el caso de la distribución de los puntajes de los participantes por la variable sociodemográfica edad, se presentan variantes con referencia a los individuos con edades entre los 32 y 40 años. En ellos se evidencian diferencias estadísticamente superiores y significativas $\left(F_{(3,780)}=2.63 ; p<.04\right)$ en cuanto a la percepción de la variable Demandas de rol como potencial generador de estrés por sobre quienes tienen entre 41 a 50 años $(p<.03)$ y 51 a 70 años $(p<.008)$. En esta interacción específica, los participantes de 32 a 40 años presentan un incremento en cuanto a la valoración de los puntajes de esta variable, pasando de una calificación media a media alta.

En la variable Desarrollo laboral, los sujetos con edades comprendidas entre los 51 y 70 años de edad presentan diferencias estadísticamente significativas e inferiores $\left(F_{(3,780)}=13.09 ; p<.0001\right)$ en cuanto a la percepción de esta como potencial generadora de estrés por sobre quienes tienen de 19 a 31 años, 32 a 40 años y 41 a 50 años (todos en un nivel $p<.0001$ y sin diferencia entre sí). La agrupación de participantes con edades entre los 51 y 70 años de edad desciende en cuanto a su puntuación general, pasando de un nivel medio a medio bajo.

Las Demandas interpersonales son percibidas como una potencial variable generadora de mayores y significativos niveles de estrés $\left(F_{(3,780)}\right.$ 


\section{TABLA 9}

\section{CORRELACIÓN ENTRE LOS ÍNDICES DE LAS VARIABLES Y EL ÍNDICE GENERAL}

\begin{tabular}{|c|c|c|c|c|c|c|c|c|}
\hline & Liderazgo & $\begin{array}{c}\text { Estruct. } \\
\text { Organiz. }\end{array}$ & $\begin{array}{c}\text { Demandas } \\
\text { rol }\end{array}$ & Desarr.Lab. & $\begin{array}{c}\text { Demandas } \\
\text { Interpers. }\end{array}$ & $\begin{array}{c}\text { Demandas } \\
\text { tarea }\end{array}$ & $\begin{array}{c}\text { Condiciones de } \\
\text { trab. }\end{array}$ & índice gral. \\
\hline Liderazgo & - & $0.72 ; .0001$ & $0.70 ; .0001$ & $0.53 ; .0001$ & $0.61 ; .0001$ & $0.46 ; .0001$ & $0.41 ; .0001$ & $0.88 ; .0001$ \\
\hline Estruct. Organiz. & & - & $0.69 ; .0001$ & $0.52 ; .0001$ & $0.63 ; .0001$ & $0.46 ; .0001$ & $0.52 ; .0001$ & $0.91 ; .0001$ \\
\hline Demandas rol & & - & $0.46 ; .0001$ & $0.54 ; .0001$ & $0.43 ; .0001$ & $0.44 ; .0001$ & $0.82 ; .0001$ \\
\hline Desarr.Lab. & & & - & $0.44 ; .0001$ & $0.32 ; .0001$ & $0.36 ; .0001$ & $0.65 ; .0001$ \\
\hline Demandas Interpers. & & & & & - & $0.41 ; .0001$ & $0.40 ; .0001$ & $0.74 ; .0001$ \\
\hline Demandas tarea & & & & & & - & $0.31 ; .0001$ & $0.59 ; .0001$ \\
\hline Condiciones de trab. & & & & & & & - & $0.59 ; .0001$ \\
\hline índice gral. & & & & & & & & - \\
\hline
\end{tabular}

Nota. Los datos presentados en cada casilla corresponden al nivel de correlación producto momento de Pearson y su respectivo grado de significancia (r; $p<.05)$.

$N=786$

$=3.63 ; p<.01)$ por parte de los sujetos con edades entre los 41 a 50 años en relación con los sujetos de 19 a 31 años y 51 a 70 años $(p<.02$ y $p<.007$ respectivamente). Esta tendencia se repite entre los sujetos entre los 32 a 40 años de edad en comparación con quienes tienen 51 a 70 años de edad $(p<.02)$. Tanto los participantes entre los 19 a 31 años de edad como los que tienen entre los 51 a 70 años de edad, en esta variable particular, descienden de un puntaje general medio alto a medio.

En cuanto al puntaje del Índice General sobre la percepción de algunas variables organizacionales como potenciales generadores de estrés, los participantes con edades entre los 32 a 40 años presentan valores significativamente superiores $\left(F_{(3,780)}=2.84 ; p<.03\right)$ que los que tienen entre 51 a 70 años $(p<.007)$, ascendiendo su calificación general a medio alto.

En el caso de quienes poseen hijos, específicamente sobre la variable Desarrollo laboral, se encontraron diferencias estadísticamente superiores $\left(F_{(4,779)}=3.51 ; p<.007\right)$ por parte de quienes no tienen hijos en comparación con quienes tienen entre 1 y 3 hijos ( $p<.01$ y $p<.001$ respectivamente). A pesar de ello, el puntaje general se mantiene en una calificación media.

A partir de la clasificación de participantes según la naturaleza de la organización, se encontraron diferencias estadísticamente superiores y significativas por parte de los empleados del sector público por sobre el sector privado en cuanto a la percepción de la Estructura organizacional y Condiciones de trabajo $\left(F_{(1,782)}=5.091 ; p<\right.$ $.02 ; F_{(1,782)}=4.49 ; p<.03$ respectivamente) como potenciales generadores de estrés. En cuanto a Demandas de rol, la puntuación tuvo un comportamiento inverso, siendo los participantes del sector privado por sobre los participantes del sector público los que lo perciben en mayor medida y con puntajes significativos $\left(F_{(1,782)}=4.84 ; p\right.$ $<.03)$.

Considerando la antigüedad en la organización para la cual laboran, se encontraron diferencias estadísticamente superiores en cuanto a la percepción de la variable Demandas de rol como potencial generador de estrés $\left(\mathrm{F}_{(3,780)}=6.29 ; p<\right.$ .0001), por parte de quienes tienen 0.5 a 5 años 


\begin{tabular}{|c|c|c|c|c|}
\hline Variable & Mínimo & Máximo & $M$ & DT \\
\hline Liderazgo & 0 & 36 & 14.08 & 9.92 \\
\hline Estructura organizacional & 0 & 52 & 24 & 12.33 \\
\hline Demandas de rol & 0 & 24 & 8.41 & 5.82 \\
\hline Desarrollo laboral & 0 & 12 & 6.05 & 3.99 \\
\hline Demandas interpersonales & 0 & 16 & 7 & 4 \\
\hline Demandas de la tarea & 0 & 16 & 7.38 & 3.52 \\
\hline Condiciones de trabajo & 0 & 12 & 4.23 & 3.11 \\
\hline Índice general & 0 & 160 & 71.03 & 34.41 \\
\hline
\end{tabular}

Fuente: Elaboración propia.

de laborar por sobre quienes tienen 5.1 a 10 años $(p<.01)$ y 20.1 a 60 años $(p<.0001)$. Asimismo, quienes tienen 10.1 a 20 años perciben mayor estrés cuanto a esta misma variable que quienes laboran desde hace 20.1 a 60 años. En el caso particular de quienes han laborado de 0.5 a 5 años, su calificación general en Demandas de rol asciende de medio a medio alto. Por otra parte, quienes han laborado entre 10.1 a 20 años perciben mayor y significativo estrés ante la variable Condiciones de trabajo $\left(F_{(3,780)}=2.78 ; p<.04\right)$ que quienes tienen de 5.1 a 10 años $(p<.0001)$ y 20.1 a 60 años $(p<.0001)$.

En cuanto a la variable Desarrollo laboral, esta es percibida por quienes tienen mayor cantidad de tiempo de laborar (20.1 a 60 años) en menor y significativa medida $\left(F_{(3,780)}=10.26 ; p<.0001\right)$ que quienes han trabajado de 0.5 a 5 años ( $p<$ .0001), 5.1 a 10 años $(p<.0001)$ y 10.1 a 20 años $(p<.0001)$, sin diferenciarse estos últimos tres rangos entre sí. Esta tendencia es equivalente en el caso del Índice general $\left(F_{(3,780)}=3.14 ; p<.02\right)$, el cual presenta niveles inferiores y significativos entre quienes han laborado de 20.1 a 60 años y quienes lo han hecho de 0.5 a 5 años $(p<.01)$ y 10.1 a 20 años $(p<.01)$. En este particular, el nivel de percepción sobre las variables organizacionales medidas como potenciales generadores de estrés asciende de un nivel medio a un nivel medio alto para los rangos 0.5 a 5 años y 10.1 a 20 años.

\section{DISCUSIÓN}

El objetivo del presente estudio fue valorar la percepción de profesionales de la administración y los negocios sobre algunas variables organizacionales como potenciales generadores de estrés laboral. Los resultados descritos refieren a una calificación media en cuanto a los niveles alcanzados por los participantes del estudio a excepción de las variables Estructura organizacional y Demandas interpersonales. Adicionalmente, variables tales como Demandas de rol, Desarrollo laboral, y Condiciones de trabajo fueron susceptibles a cambios en función de su clasificación a partir de variables tales como edad, si cuentan con hijos, naturaleza de la organización y antigüedad de laborar para la empresa.

En el caso de los puntajes alcanzados en cuanto a la variable Estructura organizacional, debe considerarse que para un funcionamiento administrativo que favorezca el logro de los objetivos de la institución, se requiere de una estructura organizativa equilibrada; en caso contrario, puede verse comprometido el alcance de los resultados esperados (Barahona, Pérez, y Cruz 2009). Adicionalmente, 
Millán y D'Aubeterre (2012), señalan que precisar y asegurar un conjunto de condiciones de trabajo que favorezcan la salud psicológica de los trabajadores previene la presencia de indicadores de salud adversos, tales como el estrés laboral.

Es relevante destacar que las diferencias presentadas dependiendo de la naturaleza del trabajo (público o privado) constituyen una variante común no solo en Costa Rica. Las características a nivel económico y político de cada sector tienen un efecto que incide en la satisfacción y compromiso con el trabajo (Baarspul y Wilderom, 2011). La burocracia que fundamenta el ejercicio público costarricense y la dificultad en cuanto la posibilidad de modificar el funcionamiento de una organización de esta naturaleza, justifica el cambio en cuanto a los niveles presentes en esta variable por parte de los consultados.

A pesar de las diferencias identificadas por la naturaleza de la empresa para la cual laboran, existe una coincidencia en cuanto a que la Estructura de la organización debe favorecer la motivación del trabajador. Lo anterior es un medio que le permite asumir responsabilidades y encaminar su conducta laboral al logro de las metas que permitirán que la empresa alcance altos niveles de eficacia y desempeño laboral (Caligiore y Díaz, 2003).

La variable Estructura organizacional tiene una relación directa con las Demandas de rol. Estas demandas se enmarcan en un contexto organizacional y consideran adicionalmente factores físicos y sociales que requieren un esfuerzo que, en condiciones adversas, puede derivar en costos a nivel psicológico (Demerouti, Bakker, Nachreiner y Schaufeli, 2001). Adicionalmente, es comprobado que el aumento de las demandas laborales está relacionado con una mayor sensación de agotamiento (Bakker, Demerouti, Taris, Schaufeli y Schreurs, 2003).

Regularmente, el trabajador con menor edad y menor tiempo de permanecer en la institución es susceptible, en mayor medida, a someterse a condiciones más exigentes en esta línea, lo cual es coincidente con los datos reportados en esta investigación. Es relevante indicar que la condición de exigencia a partir de la naturaleza de la organización varía. El incremento en cuanto al puntaje por parte de quienes laboran en el sector privado obedece a que ese sector presenta mayor movimiento en cuanto a demandas de rol, siendo este último más estable y cuenta con mayores condiciones de estructura y constancia en cuanto a labor del funcionario.

En materia de demandas interpersonales, Peiró (2009) refiere a la importancia del análisis de estas interacciones, con miras a no afectar lo referente al cumplimiento de los objetivos que la empresa tiene planteados. En un contexto organizacional en el cual el trabajador se encuentra expuesto a constante cambio, una gestión inadecuada podría incurrir en una potencial fuente de estrés.

En cuanto al Desarrollo laboral, esta variable presentó variantes relacionadas con la edad cronológica y la antigüedad en la organización (valores altos en ambos casos). Según Rentería, Fernández, Tenjo y Uribe (2009), la percepción de dificultad de crecimiento laboral en la organización tiende a afectar la salud del trabajador, su desarrollo personal y su calidad de vida. Ya en condición avanzada de edad o con una larga temporalidad en la empresa, esta situación de crecimiento pudo haberse desarrollado o reducido la posibilidad de ocurrencia, razón que justifica los valores alcanzados. En el caso de quienes tienen no tienen hijos, presentan una condición que favorece la focalización en su trabajo; por tanto, la posibilidad de que se valore que el estatus de su puesto no corresponde a sus expectativas y que la formación profesional que tienen no es compatible con las exigencias del trabajo que realizan se maximice (Aranda, Mares, Ramírez y Rojas, 2016).

Con referencia a la variable Condiciones de Trabajo, esta presentó variantes en función de la naturaleza de la organización. Ceballos, Valenzuela 
y Paravic (2014), refieren a que las condiciones laborales son fundamentales y se vinculan con otros aspectos de índole físico, social y hasta administrativo.

Cuando estas condiciones no son favorables, pueden ser promotoras de la insatisfacción laboral. Los cambios en el sector público (de cualquier naturaleza), tienen la particularidad de realizarse en un tiempo más prolongado de lo que regularmente toma en el sector privado, siendo esta una condicionante que justifica el puntaje expuesto.

\section{CONCLUSIONES}

En términos generales, la valoración de la percepción de los participantes sobre las variables indagadas como potenciales generadores de estrés ha resultado en un nivel medio (solo dos variables indicaron un nivel medio alto). Esto refiere a la importancia de la atención del alcance, considerando que su atención oportuna puede reducir efectos adversos a nivel personal y organizacional en personas que cumplan con las características de los participantes del estudio.

La Estructura organizacional fue una de las principales variables que los profesionales de la administración y los negocios perciben en mayor nivel como potenciales generadores de estrés (nivel medio alto). Los resultados de esta variable son susceptibles a la condición de naturaleza de la organización, puntuando de manera más alta en quienes laboran en sector privado por sobre quienes lo hacen en el sector público.

La edad cronológica y antigüedad en la organización de los participantes son condiciones que afectan los puntajes de Demandas de rol. Siendo las personas que poseen mayor edad cronológica y mayor tiempo de laborar las menos susceptibles a percibir esta variable como promotora de estrés. Adicionalmente, es percibida en un nivel mayor por parte de quienes laboran en el sector público que quienes laboran en el sector privado.
Quienes no tienen hijos perciben menos efecto de la variable Desarrollo Laboral como promotora de estrés por encima de quienes tienen al menos un hijo. Por otra parte, quienes tienen mayor edad cronológica y mayor cantidad de años pertenecer a la institución, participantes que si la perciben como elemento potencialmente estresante.

Las Condiciones de trabajo son percibidas como variable promotora de estrés en mayor medida por quienes laboran en el sector público por sobre quienes lo hacen en el sector privado.

\section{REFERENCIAS}

Ahola, K., Honkonen, T., Virtanen, M., Aromaa, A., \& Lönnqvist, J. (2008). Burnout in relation to age in the adult working population. Journal of Occupational Health, 50, 362-365.

Aranda, C., Mares, F., Ramírez, B., \& Rojas, N. (2016). Factores psicosociales y síntomas de estrés laboral en trabajadores del área de producción de una empresa metal-mecánica de El Salto, Jalisco. Cuadernos Hispanoamericanos de Psicología, 16(2), 5-32.

Assadi, H. (2003). Evaluation of job stress factors (organizational and managerial) among heads of physical education organizations. Acta universitatis palackianae olomucensis gymnica, 33(1), 47-55.

Baarspul, H. C., \& Wilderom, C. P. M. (2011). Do employees behave differently in public-vs private-sector organizations? Public Management Review, 13(7), 967-1002.

Bakker, A. B., Demerouti, E., Taris, T. W., Schaufeli, W. B., \& Schreurs, P.J.G. (2003). A multigroup analysis of the job demands-resources model in four home care organizations. International Journal of Stress Manage, 10(1), 16-38.

Barahona, J., Pérez, V., \& Cruz, N. (2009). Implicaciones de la organización interna sobre la eficiencia. La aplicación de la teoría de la agencia y la metodología DEA a las ONGD españolas. Cuadernos de Economía y Dirección de la Empresa, 12(40)17-46. 
Caligiore, C. \& DiazT. (2003) Clima Organizacional y Desempeño de los Docentes en la ULA. Estudio de un caso. Revista Venezolana de Gerencia, 8(24), 644656.

Carrión, M., Gutiérrez, A., \& López, F. (2014). Condiciones de trabajo psicosociales y desgaste psíquico en trabajadores de diversos sectores empresariales de Centro-Oriente, Suroccidente y región Caribe en Colombia. Salud Uninorte, 30(3), 311-322.

Chiavenato, I (2009). Comportamiento organizacional: la dinámica del éxito en las organizaciones (2a. ed). México: McGraw-Hill/Interamericana Editores, S.A. de C.V.

Ceballos, P., Valenzuela, S., \& Paravic, T. (2014) Factores de riesgos psicosociales en el trabajo: género y enfermería. Avances en Enfermería, 32(2), 271- 279.

Demerouti, E., Bakker, A. B., Nachreiner, F., \& Schaufeli, W. B. (2001). The job demands-resources model of burnout. Journal of Applied Psycholology, 86(3), 499-512.

García-Villamizar, G. E. (2007). Clima organizacional: hacia un nuevo modelo. Porik An, 12, 151-177.

Gencay, S. \& Gencay, O. A. (2011). Burnout among judo coaches in Turkey. Journal of Occupational Health, 53(5), 365-370.

Gil-Monte, P. (2011). El Síndrome de Quemado por el Trabajo. (3a.ed.). España: Pirámide.

Instituto Nacional de Estadística y Censos (INEC). (2013). Clasificación de Ocupaciones de Costa Rica (COCR2011). Costa Rica: Instituto Nacional de Estadística y Censos.

Jiménez, H. (2006). Modelo de competitividad empresarial. Umbral Científico, 9, 115-125.

Kerlinger, F. N. \& Lee, H. B. (2002). Investigación del Comportamiento. Métodos de Investigación en Ciencias Sociales (4ta. ed.). México D.F., México: McGraw-Hill Interamericana.

Millán, A. \& D'Aubeterre, M. E. (2010). El empleo y el desarrollo de instituciones positivas: una aproximación desde el marco legal, el contexto organizacional y las investigaciones relacionadas con el bienes- tar. En C. Camilli y M. E. Garassini (Eds.). Psicología positiva: Estudios en Venezuela, 336-355. Caracas: Sociedad Venezolana de Psicología Positiva.

Moreno, B. \& Báez C. (2010). Factores y riesgos psicosociales, formas, consecuencias, medidas y buenas prácticas. NIPO792-11-088-1. España: Universidad Autónoma de Madrid.

Newstrom, J. (2011). Comportamiento Humano en el Trabajo. (13a.ed.). Mexico: McGraw Hill.

Peiró, J. M. (1999). Desencadenantes del estrés laboral. España: Pirámide.

Peiró, J. M. (2009). Nuevas tendencias en la investigación sobre estrés laboral y sus implicaciones para el análisis y prevención de los riesgos psicosociales. Recuperado de https://www.revistavirtualpro. com/biblioteca/nuevas-tendencias-en-la-investigacion-sobre-estres-laboral-y-sus-implicaciones-para-el-analisis-y-prevencion-de-los-riesgos-psicosociales

Pérez, C. (2004). Técnicas de Análisis Multivariante de Datos. España: Pearson.

Podsakoff, N., Lepine, J., \& Lepine, M. (2007). Differential challenge stressor-hindrance stressor relationships with job attitudes, turnover intentions, turnover, and withdrawal behavior: A meta-analysis. Journal of Applied Psychology, 92(1), 438-454.

Rentería, J., Fernández, E., Tenjo, A., \& Uribe, A. (2009). Identificación de factores psicosociales de riesgo en una empresa de producción. Diversitas: Perspectivas en Psicología, 5(1), 161-175.

Robbins, S. \& Judge, T. (2013). Comportamiento Organizacional. México: Pearson.

Sterud, T., Hem, E., Lau, B., \& Ekeberg, Ø. (2011). A comparison of general and ambulance specific stressors: predictors of job satisfaction and health problems in a nationwide one-year follow-up study of Norwegian ambulance personnel. Journal of Occupational Medicine and Toxicology, 6(10), 6-10

Torres, R. M., Lambert, M. D., \& Lawver, R. G. (2010). Predicting Secondary Agriculture Teachers' Job Stress from Selected Personal, Family, and Work-Related 
Characteristics. Career \& Technical Education Research, 35(2), 65-79.
Unger, K. (2018). Innovación, competitividad y rentabilidad en los sectores de la economía mexicana. Gestión y Política Pública, 27(1), 3-37.

Recibido: 07 de marzo 2018

Aceptado: 05 de marzo de 2019

APÉNDICE

CUESTIONARIO PARA LA VALORACIÓN DE FACTORES GENERADORES DE ESTRÉS EN LAS ORGANIZACIONES (CUVEO)

\section{Elaborado por Diego J. Quirós}

A continuación, se presentan una serie de situaciones que ocurren en el trabajo. Por favor marque con equis ("X") la FRECUENCIA con que las siguientes situaciones SON PERCIBIDAS por su persona COMO GENERADORAS DE ESTRÉS.

Considere para su repuesta las SITUACIONES LABORALES INDIVIDUALES (NO GRUPALES, NI DE TERCEROS) vividas en el trabajo durante al menos LOS ÚLTIMOS 12 MESES (puede ser un período más prolongado, pero NO más corto). MARQUE

0 = si la situación "NUNCA" es percibida como fuente de estrés.

1 = si la situación "CASI NUNCA" es percibida como fuente de estrés.

2 = si la situación "ALGUNAS VECES" es percibida como fuente de estrés.

3 = si la situación "CASI SIEMPRE" es percibida como fuente de estrés.

4 = si la situación "SIEMPRE" es percibida como fuente de estrés.

\begin{tabular}{|c|c|c|c|c|c|}
\hline & 0 & 1 & 2 & 3 & 4 \\
\hline \multicolumn{6}{|l|}{ 1. Tomar decisiones para resolver las tareas que tengo a cargo. } \\
\hline \multicolumn{6}{|l|}{ 2. La presión que ejercen los supervisores de mi trabajo. } \\
\hline \multicolumn{6}{|c|}{ 3. La falta de apoyo personal recibido por parte de mis compañeros de departamento. } \\
\hline \multicolumn{6}{|l|}{ 4. No contar con la tecnología adecuada para hacer un trabajo de calidad. } \\
\hline \multicolumn{6}{|l|}{ 5. El que la empresa carezca de dirección y objetivos. } \\
\hline \multicolumn{6}{|l|}{ 6. La temperatura ambiental del espacio físico donde debo realizar mi trabajo. } \\
\hline \multicolumn{6}{|l|}{ 7. La inflexibilidad de los procesos organizacionales. } \\
\hline \multicolumn{6}{|l|}{ 8. La falta de comunicación con mi jefatura inmediata. } \\
\hline \multicolumn{6}{|c|}{ 9. La falta de oportunidad de ocupar un puesto distinto al que desempeño actualmente. } \\
\hline \multicolumn{6}{|c|}{ 10. La dificultad para resolver las tareas que tengo a cargo. } \\
\hline \multicolumn{6}{|c|}{ 11. Que esperen que haga más de lo que el tiempo me permite en mi rol de trabajo. } \\
\hline \multicolumn{6}{|c|}{ 12. La falta de honestidad con la que mis compañeros de trabajo se relacionan conmigo. } \\
\hline \multicolumn{6}{|c|}{ 13. No tener el conocimiento tecnológico suficiente para competir dentro de la organización. } \\
\hline \multicolumn{6}{|c|}{ 14. El que las políticas organizacionales impidan mi buen desempeño. } \\
\hline \multicolumn{6}{|l|}{ 15. El ruido presente en el espacio físico donde debo realizar mi trabajo. } \\
\hline \multicolumn{6}{|l|}{ 16. La falta de capacidad de la organización para responder a las innovaciones. } \\
\hline \multicolumn{6}{|l|}{ 17. El que mi jefatura inmediata no me respete. } \\
\hline 18. La falta de oportunidad de alcanzar ascenso en mi lugar de trabajo. & & & & & \\
\hline
\end{tabular}




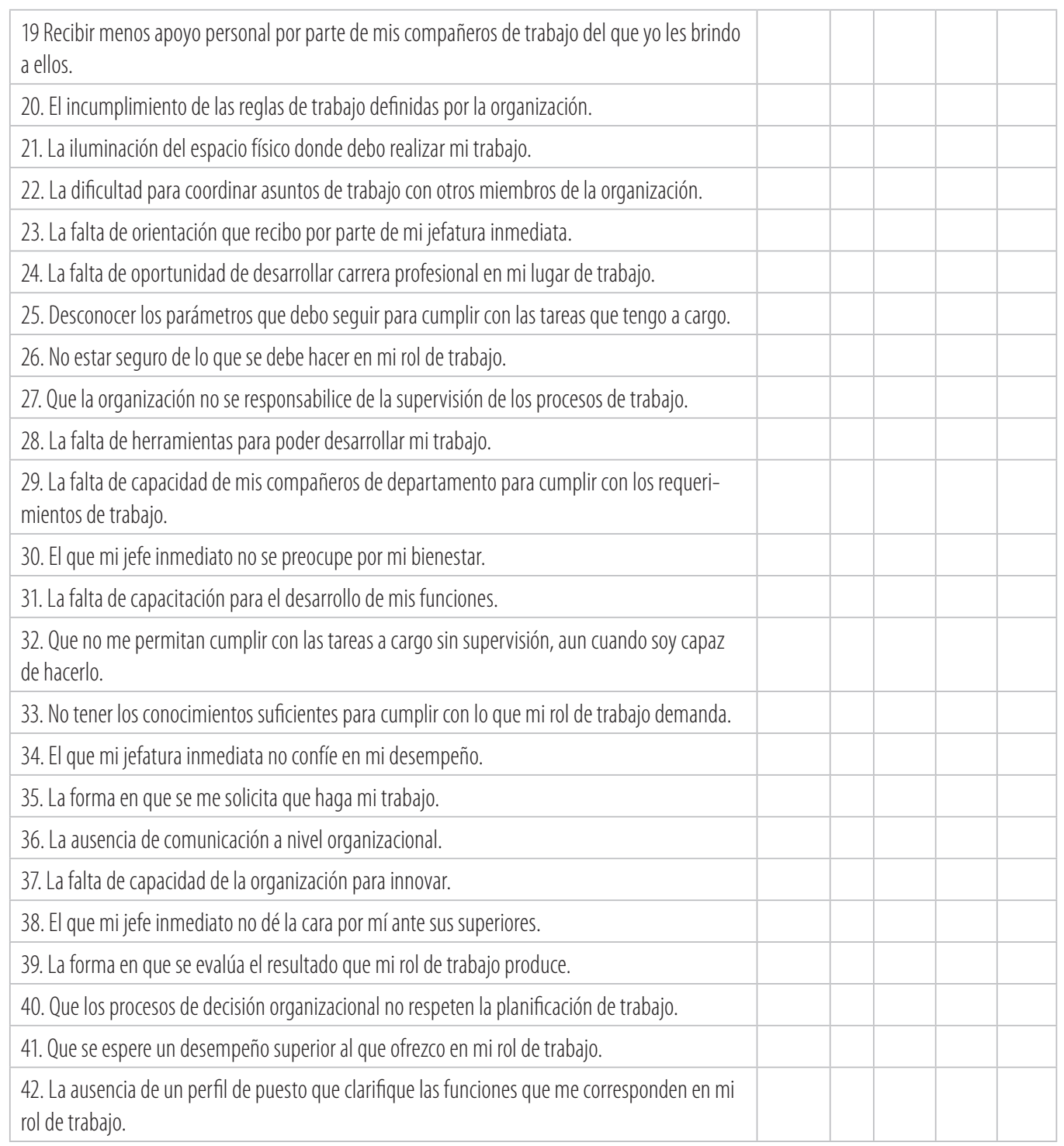

